NOTES AND CORRESPONDENCE

\title{
Seasonal Variation of the Dominant Low-Frequency Variability Observed in the Barotropic Component of the Atmosphere: A Connection to the Arctic Oscillation
}

\author{
Naomi YOKOYAMA \\ Graduate School of Life and Environmental Sciences, University of Tsukuba, Tsukuba, Japan \\ and \\ H.L. TANAKA \\ Center for Computational Sciences, University of Tsukuba, Tsukuba, Japan \\ (Manuscript received 23 April 2007, in final form 3 August 2007)
}

\begin{abstract}
In this study, EOF analyses are conducted for the barotropic component of the atmosphere in the Northern Hemisphere for each season to investigate the dominant low-frequency variability in the atmosphere. It shows that the structure of the Arctic Oscillation (AO) appears in winter and spring as a dominant mode. In summer and fall, however, the dominant modes are not the annular pattern, but show more localized structures.

The seasonal variation of the dynamical SVD-1 mode is analyzed using the climate basic states based on the singular eigenmode theory by Tanaka and Matsueda (2005) to compare with the observed EOF-1 for each season. It is found that the AO-like structure appears robustly in DJF when hyper diffusion is used in the model. The AO-like pattern obtained by observed EOF-1 appears in MAM when the viscosity is changed to the bi-harmonic diffusion. But the structures obtained as the SVD-1 in JJA and SON are different from that of the observed EOF-1. From this result, we may conclude that the AO in winter is explained by the singular eigenmode theory. However, the dominant low-frequency variability in other seasons are unlikely to be the singular eigenmode of the dynamical system explained by the SVD analysis.
\end{abstract}

\section{Introduction}

The Arctic Oscillation (AO) is a notable atmospheric phenomenon in the Northern Hemisphere in winter, which is a north-south seesaw

Corresponding author: Hiroshi L. Tanaka, Center for Computational Sciences, University of Tsukuba, 1-1-1 Tennodai, Tsukuba, Ibaraki, 3058577, Japan.

E-mail: tanaka@sakura.cc.tsukuba.ac.jp

(C) 2007, Meteorological Society of Japan of the atmospheric mass between the Arctic region poleward of $60^{\circ} \mathrm{N}$ and a surrounding zonal ring in the midlatitudes. The $\mathrm{AO}$ is defined as the primary mode of the empirical orthogonal functions (EOF-1) for the wintertime sea level pressure (SLP) anomaly in the Northern Hemisphere (Thompson and Wallace 1998: TW1998).

It may be demonstrated that the time variation in sea level pressure is dynamically equivalent to that of the barotropic component of the 
atmosphere as follows:

$\frac{\partial p_{s}}{\partial t} \simeq-\int_{0}^{p_{s}} \nabla \cdot V d p \simeq-p_{s} \nabla \cdot V_{0} \simeq \frac{p_{s}}{g h_{0}} \frac{\partial \phi_{0}}{\partial t}$.

Here, the time variation of $p_{s}$ is caused by the vertical integral of mass flux convergence $\nabla \cdot V_{0}$. The vertical integral coincides with the barotropic component of the atmosphere (noted by the subscript 0 ), which controls the time variation of the barotropic geopotential $\phi_{0}$ in a shallow water system with the depth $h_{0}$ under the gravity $g$. Hence, the AO represented by the variation in $p_{s}$ is dynamically equivalent to the variation in $\phi_{0}$. For this reason, the essential features of the $\mathrm{AO}$ are contained in the barotropic component of the atmosphere, which is governed by the $2 \mathrm{D}$ fluid mechanics for the global atmosphere.

Northern annular modes (NAM) are analyzed for the upper air atmosphere, especially for the stratosphere. Yet, those are not the barotropic component of the atmosphere, so the characteristics of the $2 \mathrm{D}$ fluid mechanics, such as inverse energy cascade, are not applied. Therefore, NAM analyzed at the sea level pressure is referred to as the AO, only which has a firmed dynamical basis of the 2D fluid mechanics governing the low-frequency variability in the extratropical atmosphere.

The study of the AO in winter is extended to summer by Ogi et al. (2004). They investigated the seasonally varying Northern Annular Mode (SV-NAM) and compared with the NAM in winter and summer. The AO in summer has a smaller meridional scale and is displaced poleward as compared to the AO in winter. The antinode on the lower-latitude side in the AO in summer is at the nodal latitude of the $\mathrm{AO}$ in winter. As the important characteristics, the AO in winter shows two centers of action over the North Pacific and the North Atlantic, whereas that in summer shows two centers of action over the northern Europe and Sea of Okhotsk.

Beside the controversial argument to recognize the $\mathrm{AO}$ as a dynamical mode or a statistical artifact (see Itoh 2002), a dynamical approach has been pursued by Tanaka and Matsueda (2005) by solving a singular mode with the smallest singular value of the linearized dynamical system, which is now referred to as the neutral mode theory (e.g., Kimoto et al. 2001; Watanabe and Jin 2004). Tanaka and Matsueda (2005) identified that for the global atmosphere, the characteristics of the singular mode similar to the AO are originated from the eigenmode of the dynamical system with nearly zero eigenvalue, i.e., singular eigenmode. Although the dynamical explanation of the AO is well presented for winter, there have been no attempts to investigate a dynamical explanation of the $\mathrm{AO}$ for other seasons.

The purpose of this study is to investigate the seasonal variation of the dominant lowfrequency variability of the atmosphere by applying the EOF analyses for the barotropic component of the atmosphere for each season. Moreover, compared with the theoretical SVD mode, we investigate whether or not the dominant low-frequency variability in each season is explained by the singular eigenmode theory.

\section{Data and method}

The data used in this study are monthly mean NCEP/NCAR reanalysis for 51 years from 1950 to 2000 in the Northern Hemisphere. The data contain horizontal winds $(u, v)$, and geopotential $\phi$ defined as every $2.5^{\circ}$ longitude by $2.5^{\circ}$ latitude grid point over 17 mandatory vertical levels from 1000 to $10 \mathrm{hPa}$. Analyses are concentrated on the barotropic component of the atmosphere since the characteristics of the surface pressure are contained in the barotropic component.

According to Tanaka (2003), the 3D representation of the spectral primitive equations on a sphere may be written as

$$
\frac{d w_{i}}{d \tau}=-i \sigma_{i} w_{i}-i \sum_{j k} r_{i j k} w_{j} w_{k}+f_{i}
$$

where $\tau$ is a dimensionless time, $\sigma_{i}$ is the eigenfrequency of the Laplace's tidal equation, $w_{i}$ is the expansion coefficient of the state variables, $f_{i}$ is the expansion coefficient of the external forcing of viscosity and diabatic heating rate, and $r_{i j k}$ is the interaction coefficient for nonlinear wave-wave interactions.

The EOF analysis is conducted for the state variable $w_{i}$ in the spectral domain of the primitive equation for each season using the longterm observational data. Therefore, the analysis domain is the entire Northern Hemisphere, and the norm used in the EOF analysis corre- 
sponds to the mass integral of the total energy (i.e., the sum of kinetic energy and available potential energy) of the hemispheric atmosphere. In this study, only the barotropic component of the $3 \mathrm{D}$ spectral model is considered to represent the simple dynamics of the low-frequency variability that indicates equivalent barotropic structure. The frictional forcing $d_{i}$ in $f_{i}$ is parameterized by the following hyper diffusion and Rayleigh friction as in Tanaka and Matsueda (2005):

$$
d_{i}=-k_{D} c_{i}^{-4} w_{i}-v_{S} w_{i}
$$

where $k_{D}=2.7 \times 10^{40} \mathrm{~m}^{8} \mathrm{~s}^{-1} /\left(2 \Omega a^{8}\right)$ is a diffusion coefficient, $c_{i}$ is a phase speed of Rossby modes given as:

$$
c_{i}=\frac{\sigma_{i}}{n} \simeq \frac{-1}{l(l+1)},
$$

where $n$ and $l$ designate zonal and meridional wavenumbers. The e-folding time for the largest meridional wavenumber $(l=20)$ for $n=0$ is one hour in this case. The linear damping coefficient $v_{S}$ is first set to zero and will be added later to shift the eigenvalues so that the system becomes singular.

Next, the nonlinear spectral equation (2) is truncated for $n=-N, N, l=0, L, m=0$, and linearized about a prescribed climate basic state in order to construct a linear system. The zonal and meridional wave truncation of the present model is equivalent to rhomboidal 20 (i.e., $L=K=20$ ) with an equatorial wall. In this study, the frictional forcing (3) is incorporated as a part of the dynamical system, and the external forcing $f_{i}$, which mainly represents baroclinic-barotropic interactions, is considered as a stochastic random process. The complex-valued linear system can be represented by a real-valued linear system, putting $\mathbf{x}=\left(w_{R i}, w_{I i}\right)$ and $\mathbf{f}=\left(f_{R i}, f_{I i}\right)$, where the subscripts $R$ and $I$ represent real and imaginary parts, respectively:

$$
\frac{d \mathbf{x}}{d \tau}=\mathbf{A x}+\mathbf{f}
$$

The real matrix $\mathbf{A}$ is determined by the given basic state. Refer to Tanaka and Kung (1989) for the detail of the evaluation of the matrix $\mathbf{A}$.

First, the eigenvalue problem (EVP) may be solved by assuming a wave-type solution for $\mathbf{x}(\tau)=\xi \exp (v \tau)$, disregarding the external forcing for eddies:

$$
\nu \mathbf{x}=\mathbf{A} \mathbf{x} .
$$

In general, the eigenvalues are complex due to the barotropic instability of the non-zonal basic state. As a special case, standing eigenmodes appear associated with real-valued eigenvalues with arbitrary signs in its fixed structure. It is those standing eigenmodes $\left(v_{I}=0\right)$ that may be important for the analysis of the Arctic Oscillation because eigenvalues with small magnitudes are searched for in present study. The most unstable standing eigenmode is the target of the AO mode in this study. The Rayleigh friction $v_{S}$ is then set to the growth rate of the most unstable mode so that the corresponding eigenvalue becomes zero, i.e., singular eigenmode, which will be identified as the SVD-1 in the next step. The assumption of the steadyness is satisfied for the singular eigenmode with zero eigenvalue.

Second, singular vectors of the linear system may be solved for neutral modes with respect to a stochastic random forcing $\mathbf{f}$ under the steady state (see Kimoto et al. 2001; Watanabe and Jin 2004).

$$
\mathbf{x}=-\mathbf{A}^{-1} \mathbf{f}=-\mathbf{V} \Sigma^{-1} \mathbf{U}^{T} \mathbf{f},
$$

where $\Sigma$ is a diagonal matrix containing singular values $s_{i}$, $\mathbf{U}$ and $\mathbf{V}$ are orthonormal matrices containing left and right singular vectors, respectively. Here, responding to the stochastic random forcing $\mathbf{f}$, steady solution $\mathbf{x}$ is obtained as a summation of the right singular vectors $\mathbf{v}_{i}$. Among those $\mathbf{v}_{1}$ associated with the smallest magnitude of the singular value (referred to as SVD-1) are considered as most important by the resonant behavior under the singular eigenmode theory.

\section{Result}

The EOF analysis is conducted for each season using the monthly data from 1950 to 2000 by the NCEP/NCAR reanalysis. Figure 1 illustrates the distribution of the EOF-1 evaluated for winter (DJF). The structure shows positive anomalies over the northern Pacific and the northern Atlantic and negative anomaly over the Arctic Ocean. This pattern is same as the AO pattern defined by TW1998. The structure of EOF-1 for spring (MAM) is similar to the 


\section{Barotropic Height}

EOF-1 for DJF (21.0\%)

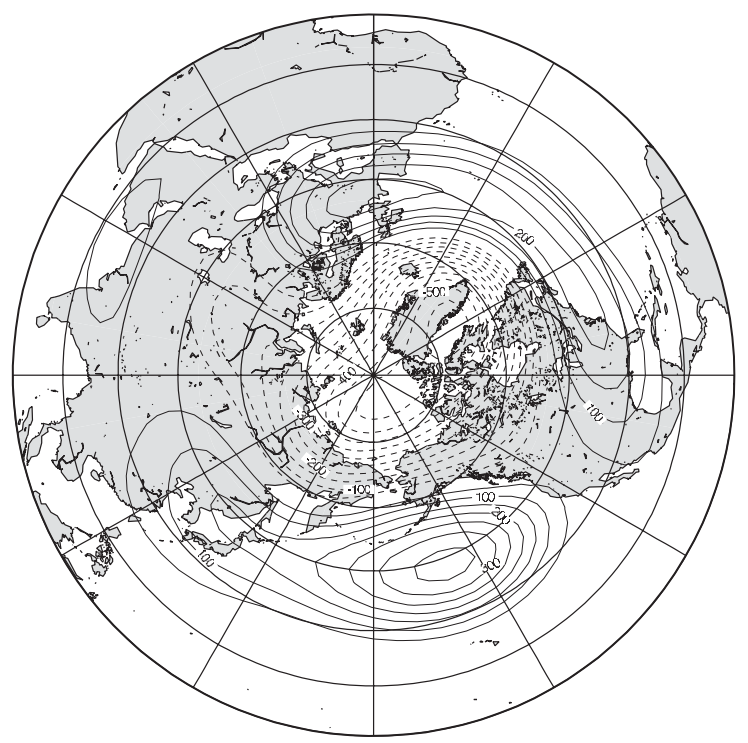

Fig. 1. Distribution of the EOF-1 evaluated for the monthly data in DJF from 1950 to 2000 by the NCEP/NCAR reanalysis.

EOF-1 for DJF, but the negative area over the Arctic is reduced northward (Fig. 2). The structure of EOF-1 for summer (JJA) loses the annular shape. It is found that the pattern is characterized by the robust positive anomaly over Europe, weak positive anomaly over the eastern Siberia, and negative anomaly over the Arctic Ocean and the northern Pacific (Fig. 3). This pattern is similar to summer SV-NAM obtained by Ogi et al. (2004). However, the negative anomaly over the northern Pacific is stronger than the summer SV-NAM. The structure derived by EOF-1 for fall (SON) is characterized by synoptic-scale waves; positive anomalies are seen from northern Pacific to Eurasia, from Europe to eastern America, and negative anomaly is seen through northern Siberia, the Arctic, and northern America (Fig. 4). The structures of EOF-1 for summer (JJA) and fall (SON) represent no annular pattern.

Next, the SVD analyses are conducted for each season using the climate basic states to compare with the observed EOF-1. First, the ei-

\section{Barotropic Height}

EOF-1 for MAM (14.4\%)

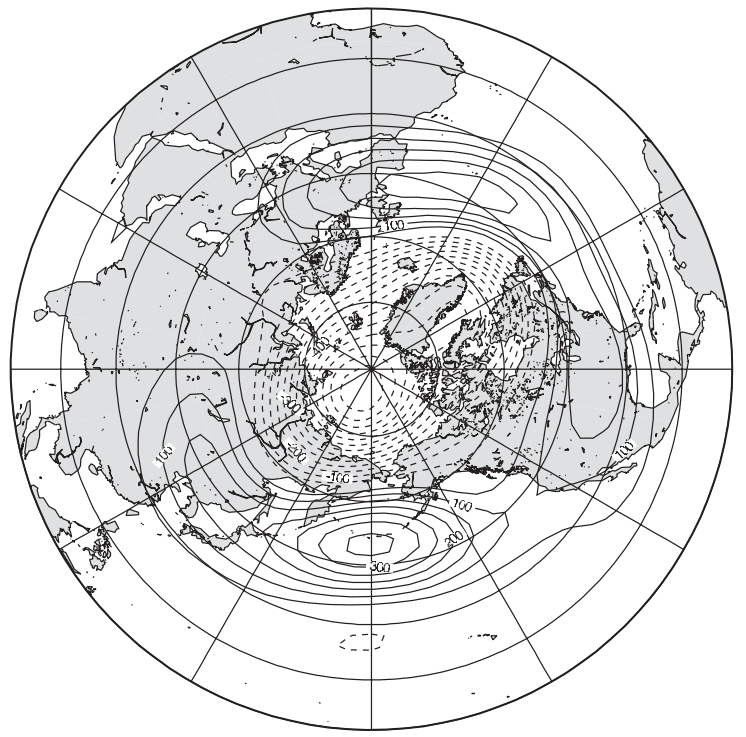

Fig. 2. Distribution of the EOF-1 evaluated for the monthly data in MAM from 1950 to 2000 by the NCEP/NCAR reanalysis.

genvalue problem (EVP) is solved for each season with the frictional forcing $d_{i}$ in $f_{i}$ parameterized by the hyper diffusion. In DJF, EVP-1 shows AO-like pattern with negative pole over the Arctic and positive pole over the Pacific and Atlantic (see Fig. 5 of Tanaka and Matsueda 2005). The dimensionless growth rate scaled by $2 \Omega$ is 0.001 . When the right amount of linear damping $v_{S}$ is introduced, the eigenvalue becomes zero. Then this mode is referred to as a singular eigenmode, which may be excited resonantly by arbitrary steady forcing. From this result, there is no doubt that the AO is explained as a dynamical normal mode of the global atmosphere.

Second, singular vectors of the linear system are solved for neutral modes (i.e., SVD analysis) with respect to a stochastic random forcing f under the steady state for each season, with the right amount of Rayleigh friction added so that the eigenvalue becomes singular. The dimensionless growth rate is 0.000 for MAM, JJA, and SON. The matrices are almost singu- 


\section{Barotropic Height}

EOF-1 for JJA (9.3\%)

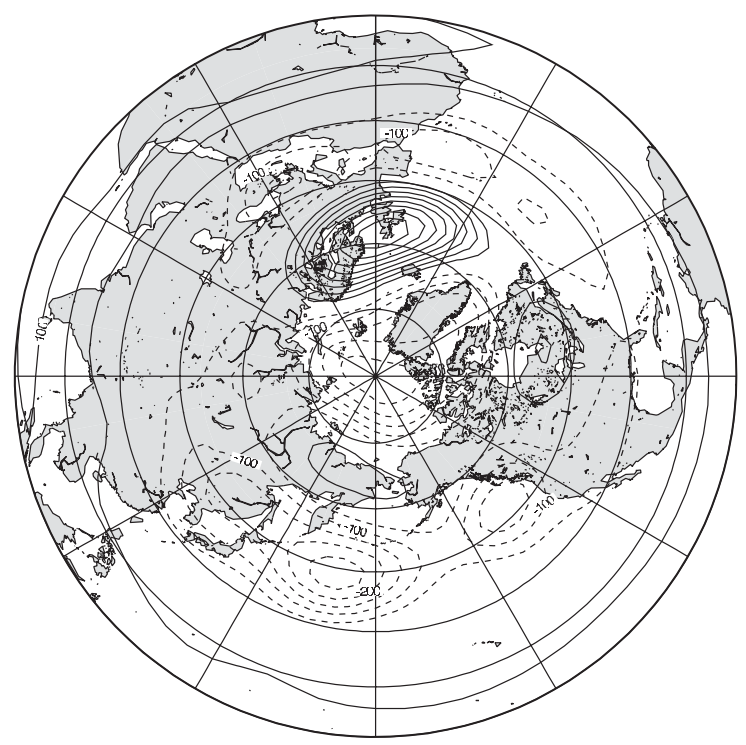

Fig. 3. Distribution of the EOF-1 evaluated for the monthly data in JJA from 1950 to 2000 by the NCEP/NCAR reanalysis.

lar from the beginning without the eigenvalue shift.

According to the result, it is found that the AO-like structure appears robustly in DJF when the frictional forcing is parameterized by the scale-dependent hyper diffusion (Fig. 5). A negative area appears over the Arctic and positive areas over the Pacific and Atlantic. The structure of the SVD-1 for DJF is identical with that of the EVP-1. Since the eigenvalue is zero for this mode, the singular value is also zero, indicating a resonant response to arbitrary steady forcing. The pattern of SVD-1 for MAM is similar to the SVD-1 for DJF, which shows a positive area over the midlatitudes and a negative area over the high latitudes (Fig. 5). It is confirmed that the node shifts southward compared with that in DJF. The patterns of SVD-1 for JJA and SON show also an annular pattern (Fig. 6). Therefore, the dynamical SVD-1 modes are different from the observed EOF-1 for MAM, JJA, and SON.

Since the obtained structures by the SVD
Barotropic Height

EOF-1 for SON (10.8\%)

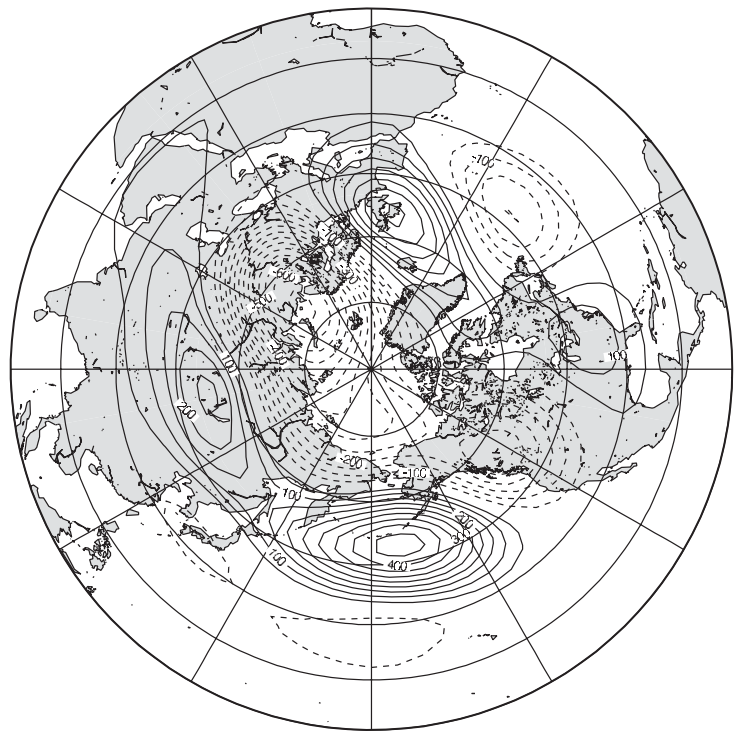

Fig. 4. Distribution of the EOF-1 evaluated for the monthly data in SON from 1950 to 2000 by the NCEP/NCAR reanalysis.

analyses are zonally symmetric, we attempted to replace the viscosity from the hyper diffusion to the bi-harmonic diffusion, and the diffusion coefficient $k_{D}=4.0 \times 10^{16} \mathrm{~m}^{4} \mathrm{~s}^{-1} /\left(2 \Omega a^{4}\right)$ is introduced, which is the same as used for the numerical experiment of the $\mathrm{AO}$ in winter (Tanaka 2003). The e-folding time of the damping for the largest meridional wavenumber $(l=20)$ for $n=0$ is three days for this case. The use of hyper diffusion tends to damp smaller scale structure, retaining the larger scale structure even for the zonal wavenumber 0 . Use of the bi-harmonic diffusion, in contrast, retains the smaller scale structure, compared with the hyper diffusion. For this diffusion, the EVP problem shows dimensionless growth rate scaled by $2 \Omega$ as 0.000 for DJF, MAM, JJA, and SON. The matrices are almost singular from the beginning without the eigenvalue shift.

Figure 7 illustrates the structures of the SVD-1 with the bi-harmonic diffusion. It is found that the dynamical SVD-1 mode in DJF is different from the observed EOF-1, and it 


\section{hyperdiffusion $(\mathrm{CDFU}=2.7 \mathrm{E} 40)$}

SVD-1 for DJF

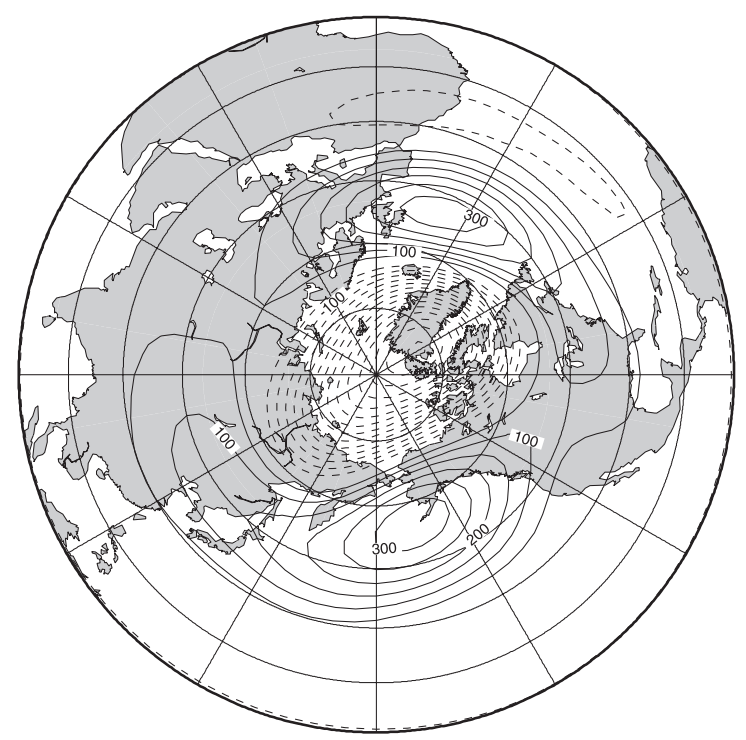

SVD-1 for MAM

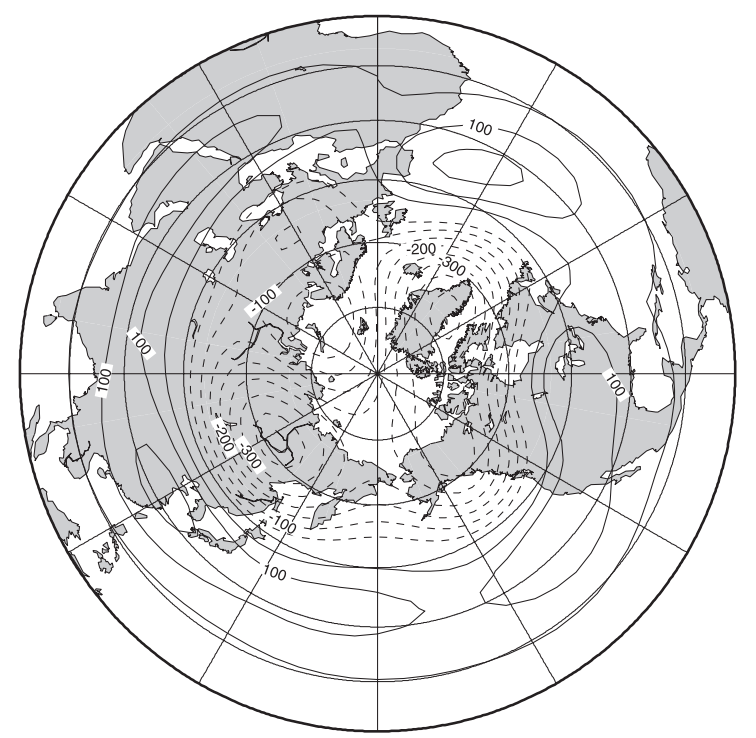

Fig. 5. Distribution of the SVD-1 evaluated for the monthly data in DJF and MAM when the viscosity is the hyperdiffusion and the diffusion coefficient $k_{D}$ is $2.7 \times 10^{40}$.

\section{hyperdiffusion $(\mathrm{CDFU}=2.7 \mathrm{E} 40)$}

SVD-1 for JJA

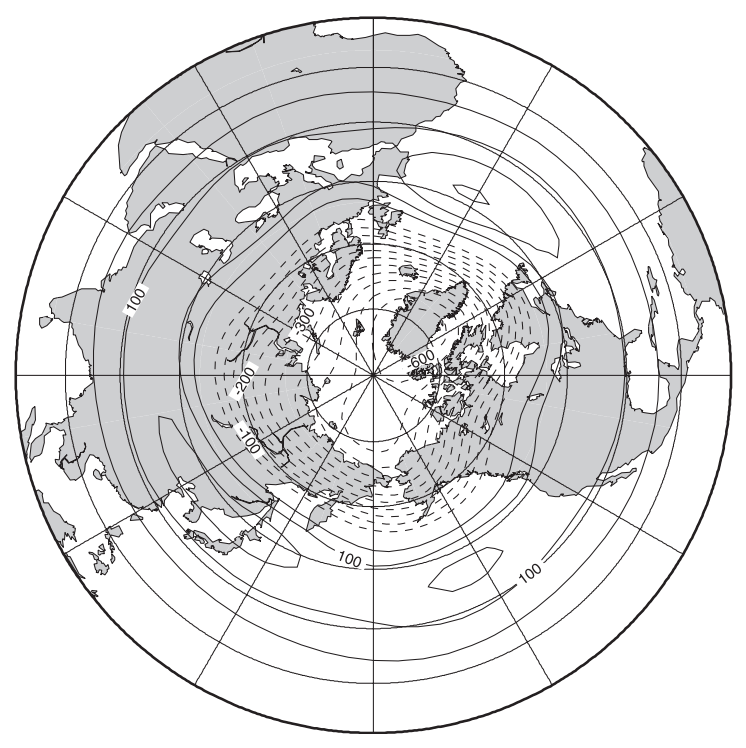

SVD-1 for SON

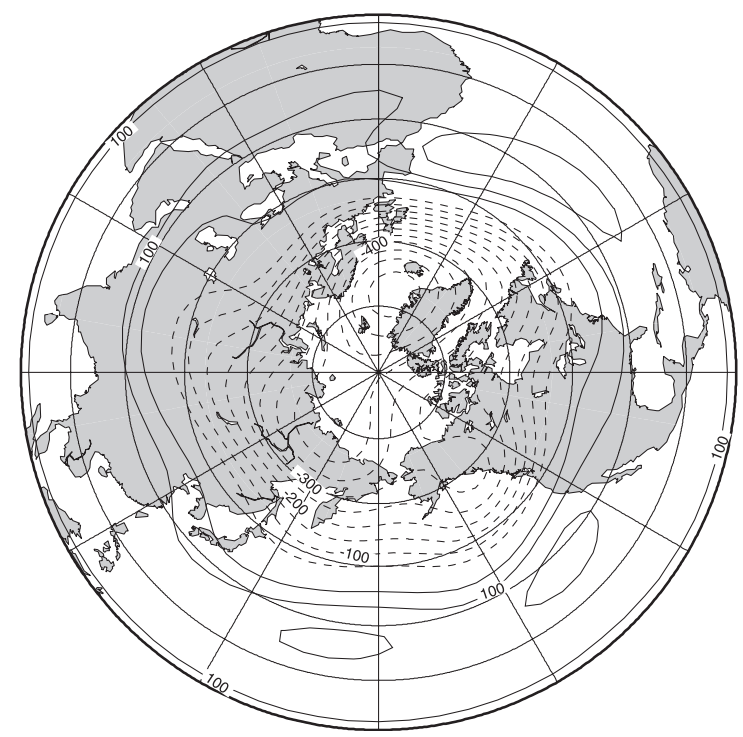

Fig. 6. Distribution of the SVD-1 evaluated for the monthly data in JJA and SON when the viscosity is the hyperdiffusion and the diffusion coefficient $k_{D}$ is $2.7 \times 10^{40}$. 


\section{biharmonic diffusion $(\mathrm{CDFU}=4.0 \mathrm{E} 16)$}

SVD-1 for DJF

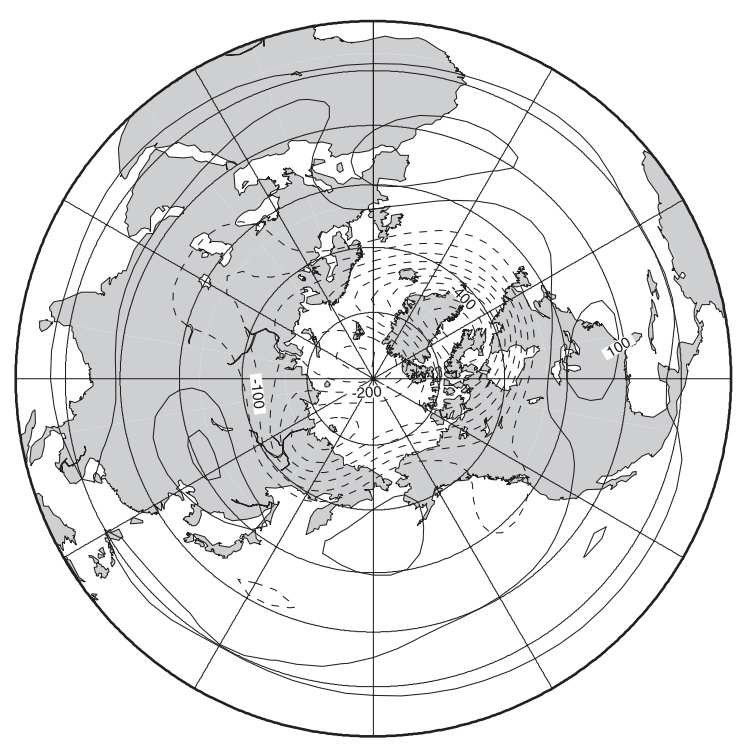

SVD-1 for MAM

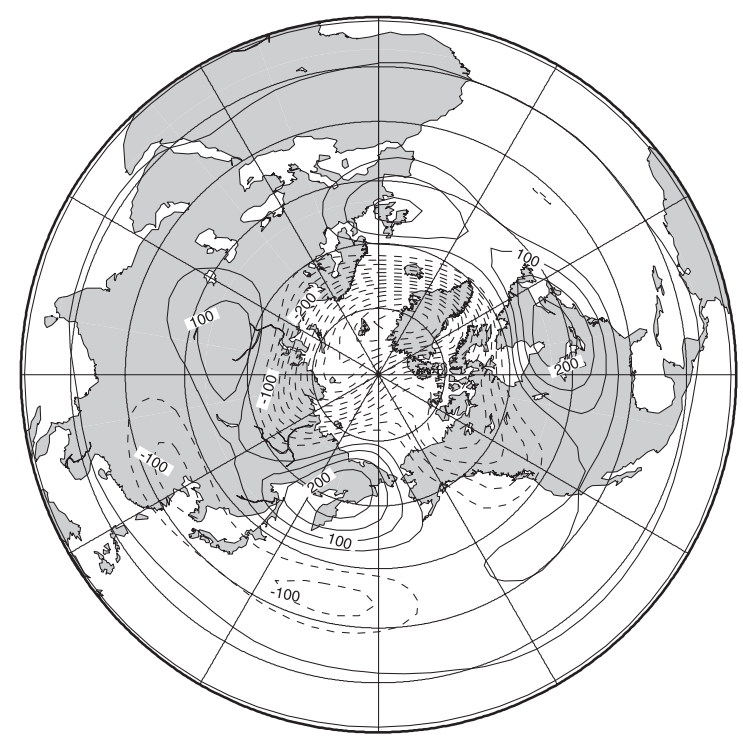

Fig. 7. Distribution of the SVD-1 evaluated for the monthly data in DJF and MAM when the viscosity is changed to the bi-harmonic diffusion and the diffusion coefficient $k_{D}$ is $4.0 \times 10^{16}$.

does not look like the AO pattern (Fig. 7). The dynamical SVD-1 mode in MAM is similar to the observed EOF-1 for the case of the biharmonic diffusion compared with that of hyper diffusion. The structure shows positive areas over the eastern America, Europe, and Russian Far East, and a negative area over the Arctic region (Fig. 7). The dynamical SVD-1 mode in JJA shows negative area over the Arctic and positive area over the midlatitudes (Fig. 8). The pattern shows annular structure, but it is different from the observed EOF-1. The pattern of dynamical SVD-1 mode in SON shows AOlike structure (Fig. 8), but it is different from the observed EOF-1. These results show that the SVD-1 is similar to the EOF-1 in MAM, while the SVD-1 is different from the EOF-1 in JJA, and SON.

\section{Concluding remarks}

In this study, the EOF analyses are conducted for the barotropic component of the atmosphere for each season. It shows that the structures of the EOF-1 in winter and spring are similar to the AO. In summer and fall, how- ever, the dominant modes are not the annular pattern, but show more localized structures. The pattern of EOF-1 in summer is different from the summer SV-NAM. It should be noted that the analysis by Ogi et al. (2004) is performed using the monthly and zonally averaged data north of $40^{\circ} \mathrm{N}$, while the entire Northern Hemisphere data are used in this study. The SV-NAM in summer appears to be sensitive to the area of the EOF analysis.

The seasonal variation of the dynamical SVD-1 mode is analyzed for the climate basic states to compare with the observed EOF-1 for each season. It is found that the AO-like structure appears robustly in DJF when hyper diffusion is used. The AO-like pattern obtained by observed EOF-1 appears in MAM when the viscosity is changed to the bi-harmonic diffusion. But the structures of the SVD-1 in JJA and SON are different from the structure of EOF-1 or higher order EOFs obtained by observational data. From this result, we may conclude that the $\mathrm{AO}$ in winter is explained by the singular eigenmode theory for the global atmosphere. However, the most dominant low-frequency 


\section{biharmonic diffusion $(\mathrm{CDFU}=4.0 \mathrm{E} 16)$}

SVD-1 for JJA

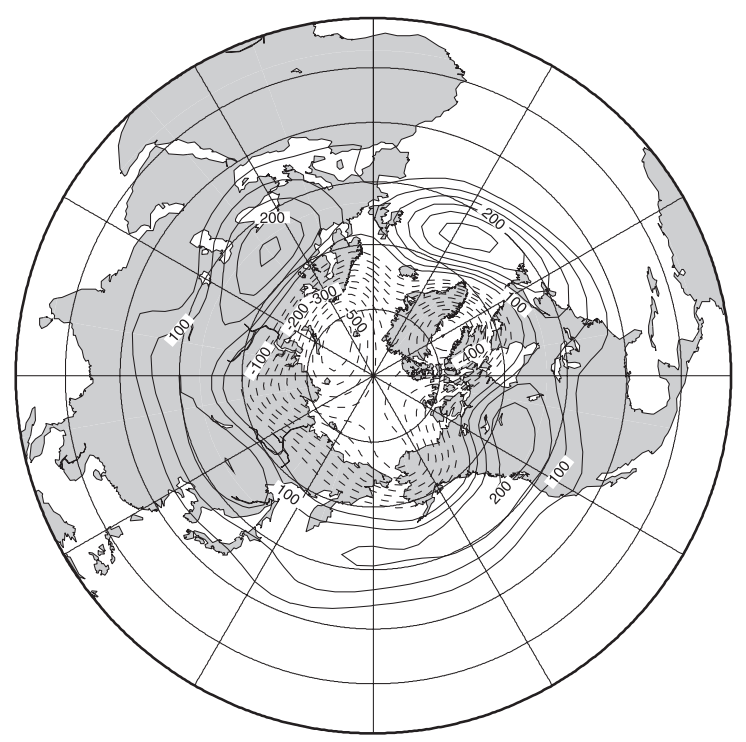

SVD-1 for SON

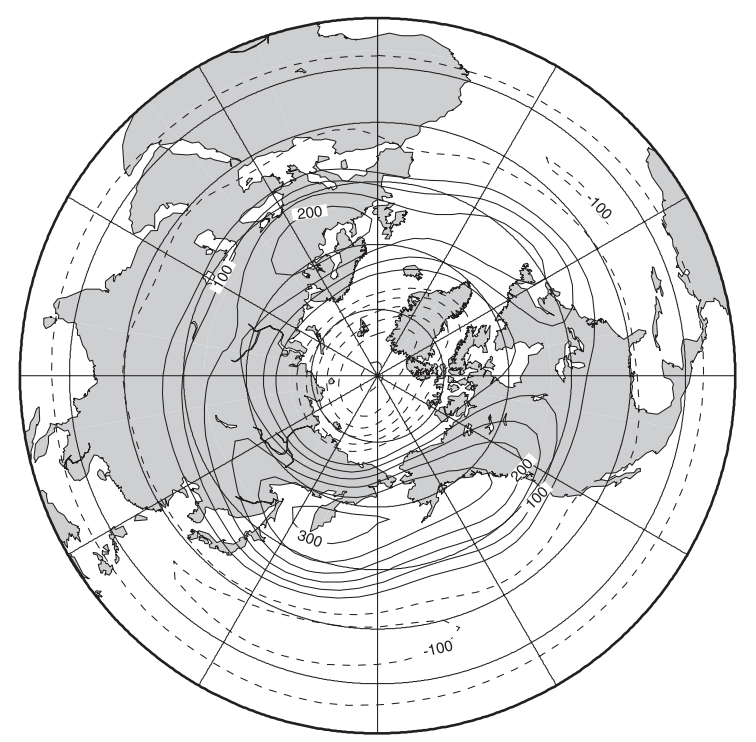

Fig. 8. Distribution of the SVD-1 evaluated for the monthly data in JJA and SON when the viscosity is changed to the bi-harmonic diffusion and the diffusion coefficient $k_{D}$ is $4.0 \times 10^{16}$.

patterns in other seasons are unlikely to be the singular eigenmode of the dynamical system. It is speculated that the EOF-1 in summer dominated by the localized structures is not generated by the internal dynamics of the system, but is forced by the prevailing baroclinicbarotropic interactions at the synoptic-scale motions.

In this study, only the standing eigenmodes are analyzed for the low-frequency variability because the singular eigenmode theory requires the eigenvalue to become zero. The periodic eigenmodes are excluded from the theory from the beginning since these modes are not excited reasonantly by any steady forcing. However, even the periodic modes may be excited if the period is sufficiently long. The present analysis can be extended to the periodic modes in future work in order to support the present conclusion.

\section{Acknowledgments}

This study is supported by Grant-in-Aids (A-18204043) of the Japan Society for the Promotion of Science. Part of the support comes from Asahi Breweries Foundation and the International Arctic Research Center (IARC/ UAF). The authors appreciate Mr. M. Matsueda for worthy discussion and Ms. K. Honda for her technical assistance.

\section{References}

Itho, H., 2002: True versus apparent Arctic Oscillation. Geophys. Res. Lett., 29, 1268, doi:10.1029/ $2001 \mathrm{GL} 012978$.

Kimoto, M., F.-F. Jin, M. Watanabe, and N. Yasutomi, 2001: Zonal-eddy coupling and neutral mode theory for the Arctic Oscillation. Geophys. Res. Lett., 28, 737-740.

Ogi, M., K. Yamazaki, and Y. Tachibana, 2004: The summer annular mode in the Northern Hemisphere and its linkage to the winter mode. J. Geophys. Res., 109, D20114, doi:10.1029/ 2004JD004514.

Tanaka, H.L., 2003: Analysis and modeling the Arctic Oscillation using a simple barotropic model with baroclinic eddy forcing. J. Atmos. Sci., 60, 1359-1379.

Tanaka, H.L. and E.C. Kung, 1989: A study of lowfrequency unstable planetary waves in realistic zonal and zonally varying basic states. Tellus., 41A, 179-199. 
Thompson, D.W.J. and J.M. Wallace, 1998: The Arctic Oscillation signature in the wintertime geopotential height and temperature fields. Geophys. Res. Lett., 25, 1297-1300.

Thompson, D.W.J. and J.M. Wallace, 2000: Annular mode in the extratropical circulation. Part 1 : month-to-month variability. J. Climate, 13, 1000-1016.

Watanabe, M. and F.-F. Jin, 2004: Dynamical prototype of the Arctic Oscillation as revealed by a neutral singular vector. J. Climate, 17, 21192138. 